\title{
Heterogeneous modelling and finite element analysis of the femur
}

\author{
Binkai Zhang, Xiang Wang ${ }^{*}$, Yangyang Yu, Jinjin Zheng.
}

Department of Precision Machinery and Precision Instrumentation, University of Science and Technology of China, Hefei, Anhui 230026, China

\begin{abstract}
As the largest and longest bone in the human body, the femur has important research value and application prospects. This paper introduces a fast reconstruction method with Mimics and ANSYS software to realize the heterogeneous modelling of the femur according to Hu distribution of the CT series, and simulates it in various situations by finite element analysis to study the mechanical characteristics of the femur. The femoral heterogeneous model shows the distribution of bone mineral density and material properties, which can be used to assess the diagnosis and treatment of bone diseases. The stress concentration position of the femur under different conditions can be calculated by the simulation, which can provide reference for the design and material selection of prosthesis.
\end{abstract}

\section{Introduction}

Hip joint and knee joint are important load-bearing parts of the human body, and also vulnerable to injury and inflammation. Joint replacement is a prevalent approach to achieve function restoration of degenerative joint diseases [1]. Therefore, the analysis of the mechanical properties of the femur is of great significance in the design and manufacture of the implantable prosthesis [2].

With the development of computer technology, finite element analysis is more and more applied to the research of bone biomechanics [3]. To obtain better analysis results, the skeleton finite elements are required to describe the geometry and material properties in detail. Compute tomography (CT) has a high contrast between bone and surrounding soft tissue and can accurately describe bone geometry. Moreover, the CT value (Hounsfield unit, $\mathrm{Hu}$ ) has an approximate linear relationship with bone density and intensity. Therefore, CT data are widely used in bone finite element modelling [4].

In this paper, in consideration of the heterogeneity of the femoral material, the Mimics and ANSYS software were used to determine the distribution of bone mineral density and material properties through CT image information. This femoral heterogeneous model can be rapidly reconstructed and provide data support and theoretical guidance for the diagnosis and treatment of bone diseases. By the finite element analysis in different simulations, we can find out the mechanical properties and weak points of the femur, which are is helpful for design and material selection of prosthesis.
Mimics (Materializes Interactive Medical Image Control System) is a medical image processing software for $3 \mathrm{D}$ design and modelling, developed by Materialise NV. Mimics is used to create 3D surface models from stacks of 2D image data for a variety of engineering applications.

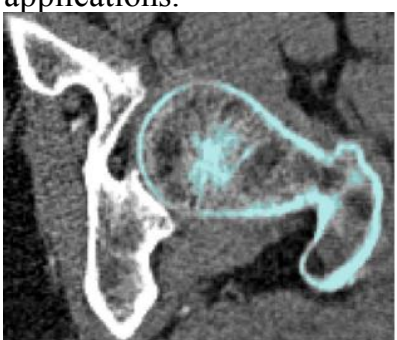

(a)

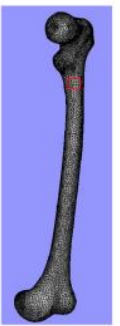

(c)

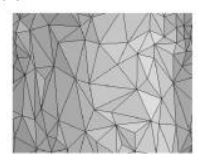

(d)

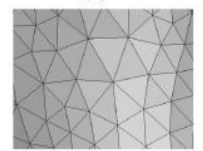

(e)

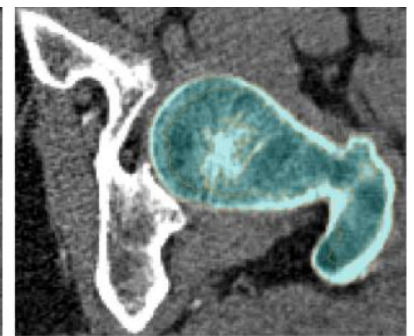

(b)

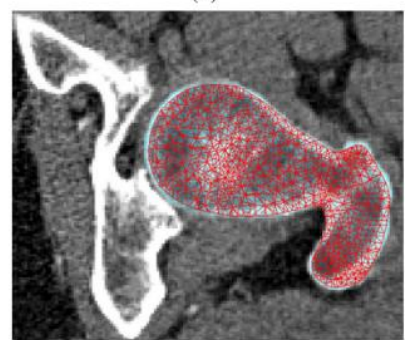

(f)
Fig. 1. 3D reconstruction of the femur by Mimics. (a)The segmentation mask after "Thresholding" and "Region Growing"; (b) The segmentation mask covered the whole femur after editing; (c)The final surface triangular mesh of the femur; (d) The partial enlargement of the surface before mesh optimization; (e) The partial enlargement of the surface after mesh optimization; (f) The cross section of the tetrahedral mesh on the image plane.

\section{Surface reconstruction}

\footnotetext{
Corresponding author: wxyf@ustc.edu.cn
} 
Here, we used Mimics software to process the CT series scanned from a female patient aged 63 years. After importing the CT data, the mask was developed to describe the femur by "Thresholding" and "Region Growing" (as shown in Fig. 1(a)). As the default threshold was set for compact bone, the mask needed to be edited to cover the whole femur (as shown in Fig. 1(b)).

Then the femoral surface triangular mesh could be reconstructed based on the mask by "3D Calculate". However, the regularisation of this initial mesh was not high as it is shown in Fig. 1(d). Therefore, the "Remesh" function was then executed to improve the regularisation by reducing triangles and smoothing the mesh, and the detail can be seen in Fig. 1(e). In this way, the femoral surface mesh was extracted from the CT series (shown in Fig. 1(c)).

\section{Heterogeneous modelling}

The accurate femoral model could be obtained by surface reconstruction using Mimics. However, in the actual finite element analysis, the distribution of the bone mineral density and mechanical properties is heterogeneous. Therefore, we reconstructed the heterogeneous model of the femur with ANSYS software.

To achieve the heterogenous modelling, the triangular mesh should be transformed to tetrahedral mesh to depict the distribution of internal materials. In ANSYS, we imported the surface triangular mesh and then performed creating tetrahedral mesh from area elements and defined the element type as "SOLID92". Then the model was exported as a tetrahedral mesh model to Mimics and the internal nodes distribution could be observed in Fig. 1(f).

After that the material property assignation should be determined according to the CT information. Through the interaction with Mimics, the internal nodes of the tetrahedral mesh can correspond to the CT image information. To achieve heterogeneous modelling of the femur, the material property of the elastic modulus, density and Poisson's ratio of model nodes could be assigned on the basis of the CT value at the corresponding location.

According to the $\mathrm{Hu}$ distribution histogram shown in Fig. 2(a), the internal material of the femur was divided into 10 types with various colours (Fig. 2(b)). The bone mineral density and elastic modulus of the femur are approximately linear with CT value $[5,6]$, and can be calculated by the following empirical formula:

$$
\begin{gathered}
\text { Density }=1041395+1017 \mathrm{Hu}, \\
\text { E-Modulus }=-388.8+5925 \text { Density, }
\end{gathered}
$$

where $H u$ indicates Hounsfield unit, and Density indicates the bone mineral density with the unit of $\mathrm{g} / \mathrm{m}^{3}$, and E-Modulus indicates the elastic modulus with the unit of $\mathrm{Pa}$.

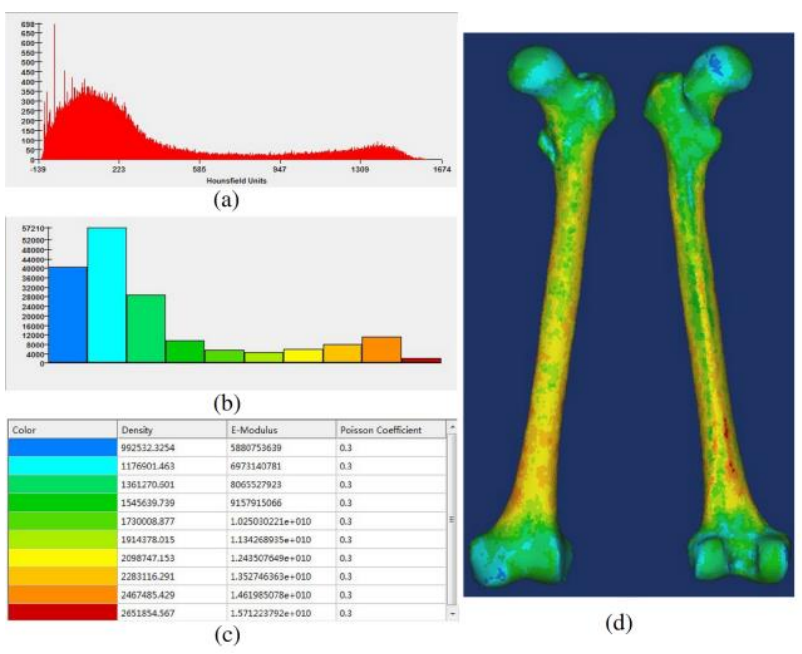

Fig. 2. The process of the femoral heterogeneous modelling. (a) $\mathrm{Hu}$ distribution histogram of the tetrahedral mesh; (b)The internal materials divided into 10 types indicated and distinguished by colour; (c)The assignment of mechanical properties of the materials; (d)Distribution of the femoral material properties from different perspectives.

As shown in Fig. 2(c), the femoral material properties such as bone mineral density, elastic modulus and Poisson's ratio were assigned in the tetrahedral mesh on the basis of $\mathrm{Hu}$ distribution, and the reconstruction of femoral heterogeneous model was completed (shown in Fig. 2(d)).

By calculating the volume of different materials, the average bone mineral density of the femur was 1.49 $\mathrm{g} / \mathrm{cm}^{3}$, which belongs to a low level. From the Fig. 2(b) and Fig. 2(d), it can be seen that the proportion of cancellous bone in the femur is the largest, which is consistent with the fact of the subject aged 63 years. Considering that the bone mineral density is an important indicator for the diagnosis of osteoporosis [7], the femoral heterogeneous model can provide data support for the diagnosis and treatment of bone diseases.

\section{Finite element analysis for the femur}

On the femoral heterogeneous model, finite element mechanical analysis can be carried out to simulate the stress distribution of the femur under different conditions. In ANSYS, we simulated the stress characteristics of the femur under the effect of gravity and horizontal impact force by defining different loads.

To analyse the effect of gravity, a vertical downward force of $500 \mathrm{~N}$ was applied to the femoral head with the displacement of the lower extremity fixed (as shown in Fig. 3(a)). On this condition, the simulation result shows that the maximum of von Miss stress is $11.8 \mathrm{MPa}$ and the maximal deformation is $2.23 \mathrm{~mm}$. From the distribution of von Miss stress (shown in Fig. 3(b)) and deformation (shown in Fig. 3(c)), it can be seen that the large stress is concentrated on the upside of the femoral shaft. 


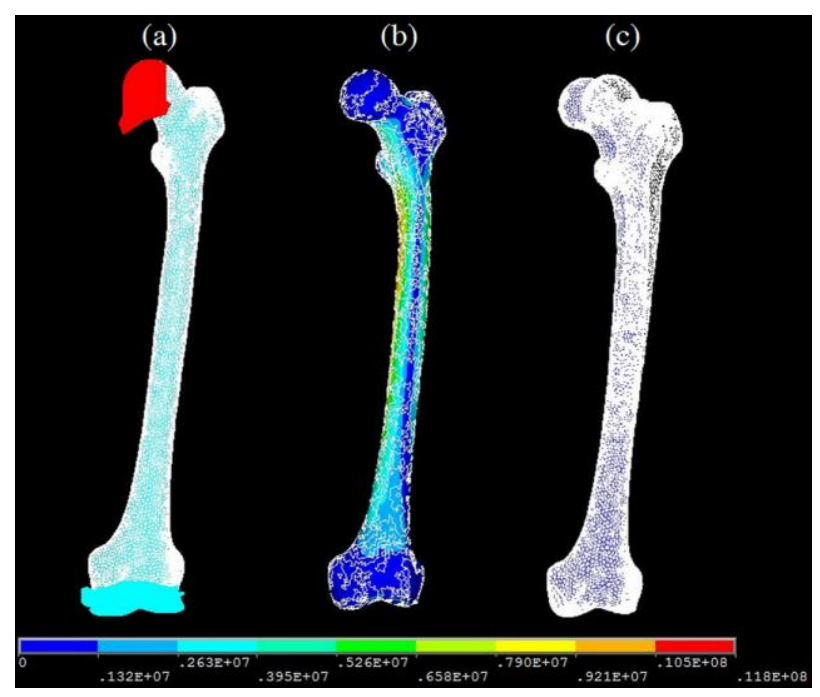

Fig. 3. Simulation when the vertical downward force applied to the femoral head with the displacement of the lower extremity fixed. (a)Applied loads; (b) Distribution of von Miss stress; (c) Deformed shape.

To simulate the effect of horizontal impact, we applied the forces in the horizontal direction of $500 \mathrm{~N}$ to three positions of the femur (shown in Fig. 4(a), Fig. 5(a) and Fig. 6(a)). The simulation results show that the maximums of von Miss stress are $15.7 \mathrm{MPa}, 12.7 \mathrm{MPa}$ and 6.3MPa (shown in Fig. 4(b), Fig. 5(b) and Fig. 6(b)), and the maximums of the deformation are $0.156 \mathrm{~mm}$, $0.283 \mathrm{~mm}$ and $0.096 \mathrm{~mm}$ (shown in Fig. 4(c), Fig. 5(c) and Fig. 6(c)), respectively. It can be seen that the maximal stress is greater on an upper position of the femur, while the maximal deformation is greater at the middle position.

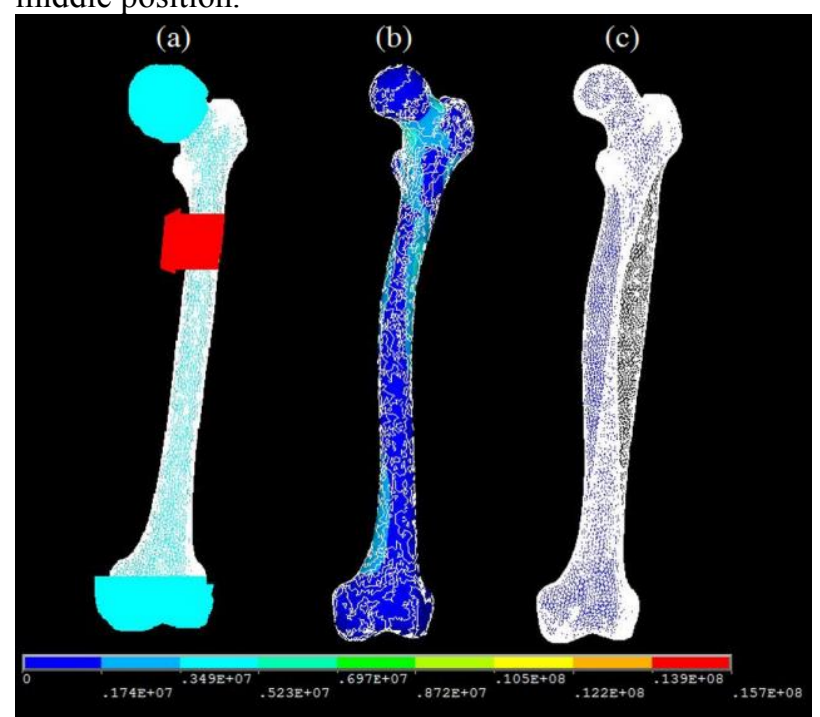

Fig. 4. Simulation when the horizontal force applied to the upper thirds of the femur with the displacement of the femoral head and the lower extremity fixed. (a)Applied loads; (b) Distribution of von Miss stress; (c) Deformed shape.

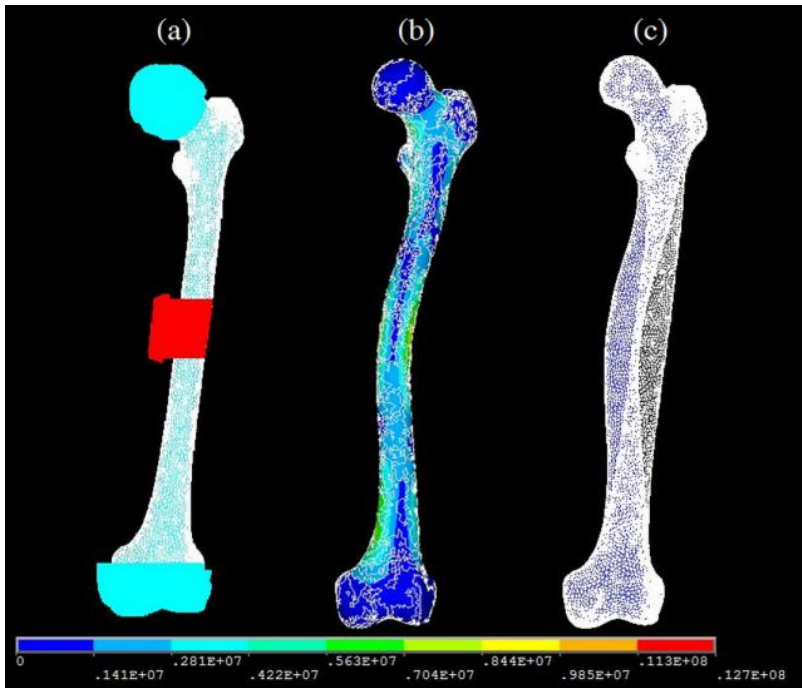

Fig. 5. Simulation when the horizontal force applied to the middle of the femur with the displacement of the femoral head and the lower extremity fixed. (a)Applied loads; (b) Distribution of von Miss stress; (c) Deformed shape.

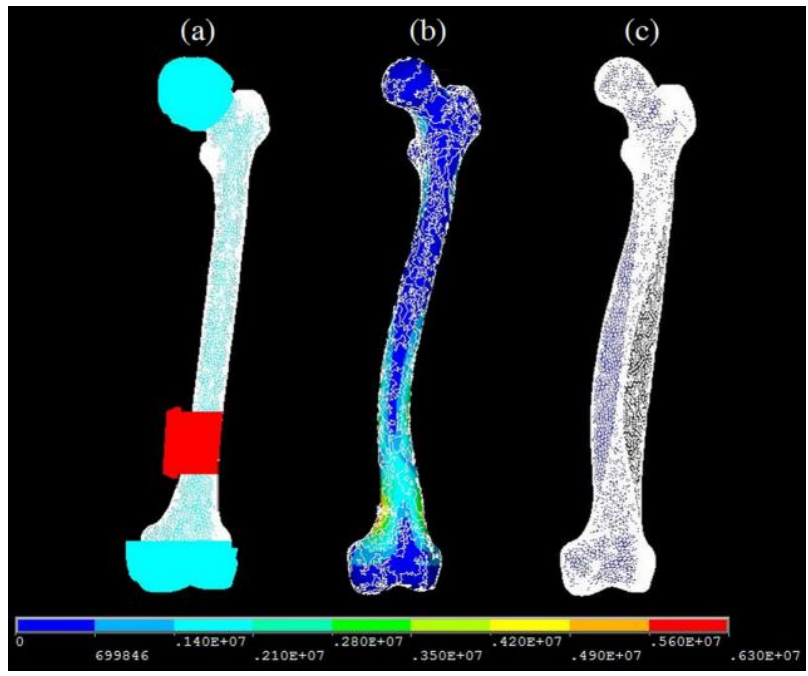

Fig. 6. Simulation when the horizontal force applied to the lower thirds of the femur with the displacement of the femoral head and the lower extremity fixed. (a)Applied loads; (b) Distribution of von Miss stress; (c) Deformed shape.

By comparing the stress distribution, it can be found that the stress concentration appears at the upper and lower sides of the femoral shaft on all the three conditions. These positions are both weak points of the femur where the fractures are prone to occur. In the design of the prosthesis, the structural strength of the stress concentration positions should be taken into consideration to avoid the periprosthetic fractures [8].

\section{Conclusion}

In this study, we proposed a method to reconstruct the femoral heterogeneous model on the basis of $\mathrm{Hu}$ distribution of CT images with Mimics and ANSYS software. Because the heterogeneous model of the femur indicates the distribution of bone mineral density and material properties, it can provide data support for the 
diagnosis and prediction for osteoporosis. In the finite element analysis, we analysed the effect of gravity and impact force by applying various loads. The stress concentration position of the femur under different conditions was calculated in the simulation, which can provide reference for the design and material selection of prosthesis.

\section{Acknowledgements}

The authors are grateful to NSFC-CAS Joint Fund [grant number U1332130], 111 Projects [grant number B07033], 973 Project [grant number 2014CB931804], and the Key Research and Development Program of Anhui [grant number 1704a0902051] for their financial support.

\section{References}

1. Peng M J Q, Chen H Y, Hu Y, et al. Finite Element Analysis of porously punched prosthetic short stem virtually designed for simulative uncemented Hip Arthroplasty. BMC Musculoskeletal Disorders, 2017, 18(1): 295.

2. Yerasimides J G. Use of the Fitmore ${ }^{\circledR}$ hip stem bone-preserving system for the minimally invasive anterior-supine approach in hip replacement. American journal of orthopedics (Belle Mead, NJ), 2010, 39(10 Suppl): 13-16.

3. Janssen D, Mann K A, Verdonschot N. Finite element simulation of cement - bone interface micromechanics: A comparison to experimental results. Journal of Orthopaedic Research, 2009, 27(10): 1312-1318.

4. Bougherara H, Zdero R, Miric $M$, et al. The biomechanics of the T2 femoral nailing system: a comparison of synthetic femurs with finite element analysis. Proceedings of the Institution of Mechanical Engineers, Part $\mathrm{H}$ : Journal of Engineering in Medicine, 2009, 223(3): 303-314.

5. Rho J Y, Hobatho M C, Ashman R B. Relations of mechanical properties to density and CT numbers in human bone. Medical engineering \& physics, 1995, 17(5): 347-355.

6. Ciarelli M J, Goldstein S A, Kuhn J L, et al. Evaluation of orthogonal mechanical properties and density of human trabecular bone from the major metaphyseal regions with materials testing and computed tomography. Journal of Orthopaedic Research, 1991, 9(5): 674-682.

7. Oden Z M, Selvitelli D M, Bouxsein M L. Effect of local density changes on the failure load of the proximal femur. Journal of Orthopaedic Research, 1999, 17(5): 661-667.

8. Beals R K, Tower S S. Periprosthetic fractures of the femur: an analysis of 93 fractures. Clinical orthopaedics and related research, 1996, 327: 238246. 